\title{
ATRIBUTOS DE SOLOS TROPICAIS E A SORÇÃO DE IMAZAQUIN
}

\author{
Jussara Borges Regitano1*; Luís Reynaldo Ferracciú Alleoni ${ }^{2,3}$; Valdemar Luiz Tornisielo \\ ${ }^{1}$ Lab. de Ecotoxicologia - USP/CENA, C.P. 96 - CEP: 13400-970 - Piracicaba, SP. \\ ${ }^{2}$ Depto. de Solos e Nutrição de Plantas - USP/ESALQ, C.P. 9 - CEP: 13418-900 - Piracicaba, SP. \\ ${ }^{3}$ Bolsista CNPq. \\ *Autor correspondente <regitano@cena.usp.br>
}

RESUMO: O imazaquin é um herbicida com caráter ácido, de uso intensivo no Brasil para controlar plantas daninhas associadas à cultura da soja. Estudou-se a sorção do imazaquin e a sua relação com a CTC, o pH e os teores de carbono orgânico $\left(C_{\text {org }}\right)$ e argila de onze amostras de solos tropicais. Para a obtenção das isotermas de sorção, soluções de imazaquin nas concentrações de 0,53;0,88; 1,45; 2,71 e 5,20 mg mL $\mathrm{m}^{-1}$ foram preparadas em $\mathrm{CaCl}_{2} 0,005 \mathrm{~mol} \mathrm{~L}^{-1} \mathrm{com}$ a adição de um traçador radioativo $\left({ }^{14} \mathrm{C}\right.$-imazaquin). $\mathrm{O}$ coeficiente de sorção $\left(\mathrm{K}_{\mathrm{f}}\right)$ foi calculado a partir da equação linearizada de Freundlich. A sorção do imazaquin variou de baixa a moderada $\left(\mathrm{K}_{\mathrm{f}}<3,0\right)$, exceto no Gleissolo. $\mathrm{O}$ teor de $\mathrm{C}_{\text {org }}$ e o $\mathrm{pH}$ da solução foram os principais atributos do solo correlacionados com a sorção do imazaquin. A inclusão do teor de argila na análise de regressão aumentou em $20 \%$ a precisão do modelo. O imazaquin apresenta maior disponibilidade e, portanto, maior potencial de lixiviação em solos com valores de $\mathrm{pH}$ acima de 6 e com baixos teores de $\mathrm{C}_{\text {org }}$ e argila.

Palavras-chave: $\mathrm{K}_{\mathrm{f}}$, retenção, herbicida, correlação, carbono orgânico, pH

\section{TROPICAL SOIL ATTRIBUTES AND THE SORPTION OF IMAZAQUIN}

\begin{abstract}
Imazaquin is an acid herbicide widely used in Brazil to control weeds associated to the soybean crop. The purpose of this research was to study imazaquin sorption and its relation to $\mathrm{CEC}, \mathrm{pH}$ and organic carbon (OC) and clay contents of eleven tropical soil samples. In order to establish the sorption isotherms, imazaquin solutions at concentrations of $0.53,0.88,1.45,2.71$, and $5.20 \mathrm{mg} \mathrm{mL}^{-1}$ were prepared in $0.005 \mathrm{~mol}$ $\mathrm{L}^{-1} \mathrm{CaCl}_{2}$ solution with the addition of radiolabeled tracer $\left({ }^{14} \mathrm{C}\right.$-imazaquin). The sorption coefficients $\left(\mathrm{K}_{\mathrm{f}}\right)$ were calculated according to the linear shape of the Freundlich isotherm equation. Imazaquin sorption ranged from low to moderate $\left(\mathrm{K}_{\mathrm{f}}<3.0\right)$, except for the Aquox soil. OC content and $\mathrm{pH}$ were the soil attributes that best correlated to imazaquin sorption. The incorporation of clay content in the regression analysis increased the model's precision by $20 \%$. Imazaquin shows higher availability and, therefore, higher leaching potential when applied to soils with $\mathrm{pH}$ higher than 6 and low contents of $\mathrm{OC}$ and clay.

Key words: $\mathrm{K}_{\mathrm{f}}$, retention, herbicide, correlation, organic carbon, $\mathrm{pH}$
\end{abstract}

\section{INTRODUÇÃO}

A adoção de práticas conservacionistas de manejo de solo no Brasil, como o sistema de semeadura direta ocasiona alterações no processo de produção agrícola. Uma vez instalado o sistema, o revolvimento do solo passa a ser bem menor e, com isso, o preparo deixa de ser utilizado para o controle das plantas daninhas. Como conseqüência, tem aumentando a dependência do uso de herbicidas, principalmente em áreas de plantio de soja. Nos últimos dez anos, cerca de US $\$ 800$ milhões por ano foram gastos com o consumo de herbicidas no país, sendo que somente a cultura da soja absorveu praticamente metade deste montante. Desta forma, torna-se fundamental entender o comportamento e o destino destes herbicidas no ambiente.

O imazaquin (ácido 2-[4,5dihidro-4-metil-4-(1metiletil)-5-oxo-1H-imidazol-2-il]-3-quinolino-carboxílico) é um herbicida de uso intensivo no Brasil, para controle de plantas daninhas associadas à cultura da soja, sendo aplicado em pré-plantio incorporado e em préemergência (Bhalla et al., 1991). Sua molécula contém dois grupos funcionais ionizáveis: um grupo carboxílico (ácido fraco, $\mathrm{pK}_{\mathrm{a}}=3,8$ ) e um grupo quinolina (base fraca, $\left.\mathrm{pK}_{\mathrm{a}}=2,0\right)$ (Stougaard et al., 1990) (Figura 1).

$\mathrm{Na}$ faixa de $\mathrm{pH}$ mais comum dos solos tropicais agricultáveis $\left(5,0\right.$ a 6,5 , na qual $\left.\mathrm{pH}>\mathrm{pK}_{\mathrm{a}}\right)$, o imazaquin comporta-se predominantemente como um herbicida ácido, dissociando-se à forma de ânion orgânico. Como a maioria dos solos apresenta carga elétrica líquida negativa, ocorre baixa sorção do imazaquin aos seus colóides, devido às forças eletrostáticas repulsivas (Loux et al., 1989; Regitano et al., 2000). Por este motivo, os herbicidas ácidos são normalmente menos sorvidos aos colóides do solo do que os herbicidas neutros ou básicos. Quando o pH da solução do solo está próximo ao $\mathrm{pK}_{\mathrm{a}}$ do herbicida, ocorre protonação do imazaquin, que passa para a forma neutra. Nessa condição, as interações hidrofóbicas com a matéria orgânica do solo passam a predominar, e a sorção aumenta. 
Para solos de clima temperado, a sorção do imazaquin está positivamente correlacionada com o teor de matéria orgânica e negativamente com $\circ \mathrm{pH}$ da solução do solo (Loux et al., 1989; Regitano et al., 1997). Regitano et al. (1997) observaram, para seis solos com cargas dependentes de $\mathrm{pH}$ e doze com cargas permanentes, que o principal mecanismo envolvido na sorção de imazaquin foi a partição hidrofóbica. Entretanto, nos sítios do solo com carga positiva, como na superfície dos óxidos de Fe e Al, houve expressiva sorção do produto, devido a mecanismos de troca aniônica e/ou formação de ligantes, principalmente em solos com baixo teor de carbono orgânico $\left(\mathrm{C}_{\text {org }}\right)$. Vários trabalhos mostram a importância destes mecanismos de ligação para outras moléculas orgânicas aniônicas (Grover, 1971; Huang et al., 1977; Goetz et al., 1986; Nicholls \& Evans, 1991; Ali \& Dzombak, 1996; Baskaram et al., 1996; Sannino et al., 1997; Evanko \& Dzombak, 1998; Regitano et al., 2000).

Loux et al. (1989) e Regitano et al. (1997) investigaram a contribuição de alguns atributos do solo $\left(\mathrm{pH}, \mathrm{C}_{\text {org }}\right.$, argila e CTC) na sorção do imazaquin em amostras de sedimentos e solos formados sob clima temperado e observaram que $\mathrm{o} \mathrm{pH}$ e o teor de $\mathrm{C}_{\text {org }}$ foram os principais atributos do solo relacionados à sorção do imazaquin. Em ambientes com clima tropical, entretanto, as condições de alta temperatura e precipitação, quando associadas com boa drenagem, favorecem o intemperismo e, consequentemente, a formação de solos com acúmulo de minerais de argila do tipo 1:1 e óxidos de Fe e Al. No Brasil, por exemplo, os Latossolos, que correspondem a esta classe de solos, compreendem cerca de 50 a $60 \%$ do território.

O objetivo deste trabalho foi avaliar correlações entre a sorção do imazaquin e os valores de $\mathrm{pH}, \mathrm{CTC}$, superfície específica (SE), argila e $\mathrm{C}_{\text {org }}$ de onze amostras de solos brasileiros com diferentes atributos químicos, físicos e mineralógicos, além de estabelecer as relações entre os valores dos coeficientes de sorção $\left(\mathrm{K}_{\mathrm{f}}\right)$, obtidos a partir da isoterma de Freundlich e os parâmetros $\mathrm{pH} / \mathrm{C}_{\text {org }}$, $\mathrm{pH} /$ argila e $\mathrm{pH} /\left(\mathrm{C}_{\text {org }} \times\right.$ argila $)$.

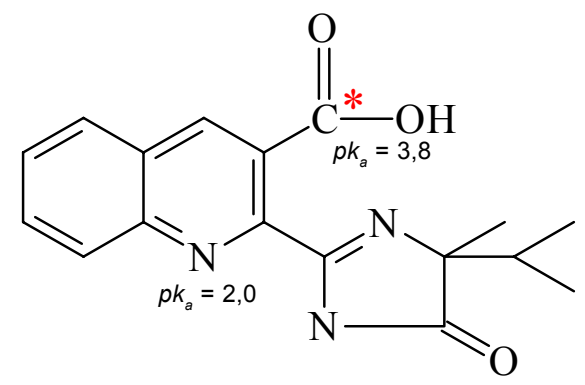

Massa molar: $311,3 \mathrm{~g} \mathrm{moL}^{-1}$

Solubilidade em água: $60 \mathrm{mg} \mathrm{L}^{-1}\left(25^{\circ} \mathrm{C}, \mathrm{pH} 3,0\right)^{-1}$

Figura 1 - Estrutura molecular do imazaquin.

\section{MATERIAL E MÉTODOS}

O estudo foi realizado em sete amostras de solos altamente intemperizados [Neossolo Quartzarênico (RQ), Nitossolo Vermelho eutroférrico (NVef), Latossolo Vermelho distroférrico (LVdf), Latossolo Vermelho distrófico $(\mathrm{LVd})$, Latossolo Vermelho-Amarelo distrófico (LVAd), Latossolo Amarelo ácrico (LAw), Latossolo Vermelho acriférrico (LVwf)] e em duas amostras de solos menos intemperizados [Vertissolo Ebânico órtico (VEo) e Gleissolo Háplico Tb distrófico (GXbd)], coletados na camada superficial $(0,0-0,2 \mathrm{~m})$. Nos solos ácricos, também foram coletados amostras subsuperficiais ( $\mathrm{LAw}^{\prime}$ e LVwf', 1,0 - 1,4 m), na maior expressão do horizonte $B$, pois nesta profundidade o balanço de cargas foi positivo. As primeiras amostras tinham grande variação nos valores de $\mathrm{C}_{\text {org }}$, argila, CTC e óxidos de ferro, de modo a abranger a maior parte dos solos brasileiros cultivados. O Vertissolo foi escolhido por apresentar constituição mineralógica diferente dos demais, com predomínio de argila expansíveis. O Gleissolo apresentava alto teor de $\mathrm{C}_{\text {org }}$ e baixo $\mathrm{pH}$, e é um dos solos exigidos pelo IBAMA (Instituto Brasileiro do Meio Ambiente e dos Recursos Naturais Renováveis), para o registro de produtos fitossanitários no Brasil, juntamente com o Latossolo Vermelho e o Latossolo VermelhoAmarelo.

As amostras de solo foram secas ao ar, peneiradas $(<2 \mathrm{~mm})$ e armazenadas. As análises foram realizadas no Departamento de Solos e Nutrição de Plantas, da Escola Superior de Agricultura "Luiz de Queiroz", da Universidade de São Paulo. Para a análise granulométrica, foi utilizado o método do densímetro, enquanto que o alumínio trocável, os cátions trocáveis e o ferro total foram extraídos com KCl $1 \mathrm{~mol} \mathrm{~L}^{-1}$, resina trocadora de íons $\mathrm{e}_{2} \mathrm{SO}_{4} 18 \mathrm{~mol} \mathrm{~L}^{-1}$, respectivamente (Camargo et al., 1986). A acidez potencial $(\mathrm{H}+\mathrm{Al}$ ) foi estimada com o uso do tampão SMP. O teor de carbono total, o qual foi assumido ser totalmente de origem orgânica, foi determinado por combustão, enquanto que a superfície específica foi determinada pelo método da retenção do éter monoetílico de etilenoglicol (Cihacek \& Bremner, 1979). Para remover a água durante a determinação, as amostras foram secas em estufa a $110^{\circ} \mathrm{C}$ por $24 \mathrm{~h}$, em lugar do pentóxido de fósforo (Ratner-Zomar et al., 1983).

Para a obtenção das isotermas de sorção, soluções de imazaquin nas concentrações de 0,53, 0,88, $1,45,2,71$ e $5,20 \mathrm{mg} \mathrm{mL}^{-1}$ foram preparadas em $\mathrm{CaCl}_{2}$ $0,005 \mathrm{~mol} \mathrm{~L}^{-1}$, com atividade radioativa correspondente a $0,17 \mathrm{kBq} \mathrm{mL}^{-1}$. Posteriormente, $2 \mathrm{~g}$ de solo e $5 \mathrm{~mL}$ de cada solução foram adicionados em tubos de centrífuga $(50 \mathrm{~mL})$, em triplicata. Os tubos foram colocados em agitador rotatório horizontal (140 rpm) por $24 \mathrm{~h}$, para homogeneização das amostras. Após o equilíbrio, os tubos foram centrifugados durante $20 \mathrm{~min}(1700 \mathrm{~g})$. Na seqüência, alíquotas de $1 \mathrm{~mL}$ do sobrenadante foram 
retiradas para determinação da concentração de imazaquin em equilíbrio na solução, por espectrometria de cintilação líquida. Em estudo preliminar, observou-se que toda a radioatividade encontrada correspondeu ao produto original, sendo possível, a quantificação do imazaquin em solução. A quantidade de imazaquin sorvida ao solo foi calculada pela diferença entre a concentração inicial e a concentração de equilíbrio. Os valores dos coeficientes de sorção $\left(K_{f}\right)$ foram obtidos a partir da equação linearizada de Freundlich: $\log S=\log$ $\mathrm{K}_{\mathrm{f}}+\mathrm{N} \log \mathrm{C}_{\mathrm{e}}$, em que $\mathrm{S}=$ concentração de imazaquin sorvido, $\mathrm{N}=$ declividade da curva e $\mathrm{C}_{\mathrm{e}}=$ concentração de equilíbrio. Estimou-se o valor do coeficiente de partição $\left(\mathrm{K}_{\mathrm{d}}\right)$, calculado quando presume-se linearidade $(\mathrm{N}=1)$ para a equação de Freundlich. Esta inferência é razoável, pois em vários experimentos relatados na literatura o valor de $\mathrm{N}$ variou de 0,83 a 0,99 , sendo as curvas próximas a linearidade.

\section{RESULTADOS E DISCUSSÃO}

A sorção do imazaquin nos solos variou de baixa a moderada, pois $K_{f}$ foi sempre menor ou igual a 3,0 (Koskinen \& Harper, 1990), à exceção do GXbd (TABELA 2). No valor de $\mathrm{pH}$ original do solo, a maioria das moléculas de imazaquin estava na forma aniônica $\left(\phi_{a}\right.$, TABELA 1), sendo repelidas pelos sítios com cargas negativas dominantes na superfície destes solos intemperizados. Paralelamente, a solubilidade do imazaquin aumenta abruptamente à medida que $\mathrm{opH}$ ultrapassa $\circ \mathrm{pK}_{\mathrm{a}}$ da molécula, diminuindo assim o coeficiente de partição entre o octanol e a água (por exemplos, $\mathrm{K}_{\text {ow }}=4,3$ a pH 4,0 e $\mathrm{K}_{\text {ow }}=0,03$ a pH 7,0), o qual simula a força das interações hidrofóbicas com a fração orgânica do solo (Stougaard et al., 1990). No $G X b d$, entretanto, o valor discrepante de $K_{f}$ foi devido tanto ao baixo valor de $\mathrm{pH}$ da solução solo, quanto ao elevado teor de $\mathrm{C}_{\text {org }}$, que são responsáveis pelo aumento na proporção de moléculas neutras e pelos sítios de interações hidrofóbicas, respectivamente.

A sorção do imazaquin depende da especiação da molécula, sendo influenciada tanto por interações hidrofóbicas com a matéria orgânica do solo, quanto por interações não-hidrofóbicas com a fração mineral do solo (Regitano et al., 1997). Em solos com baixo teor de $C_{\text {org, }}$ a fração mineral não se apresenta recoberta pelo húmus do solo, mas sim por minerais acessórios, que podem contribuir com a sorção de moléculas ionogênicas orgânicas (Stevenson, 1976; Khan et al., 1979; Hasset et al., 1981; Baskaran et al., 1996; Regitano et al., 1997).

O coeficiente de sorção $\left(K_{f}\right)$ do imazaquin correlacionou-se positivamente com o teor de $\mathrm{C}_{\text {org }}$, a CTC e a SE do solo, e negativamente com o pH da solução do solo (TABELA 3). A influência da CTC e da SE na sorção do imazaquin $\left(\mathrm{K}_{\mathrm{f}}\right)$ deve ser interpretada como efeito indireto do teor de $\mathrm{C}_{\text {org }}$, uma vez que estes parâmetros apresentaram correlações entre si $\left(r=0,92^{* *}\right.$; $0,76^{* *}, 0,82^{* *}$ para as interações CTC x $\mathrm{C}_{\text {org }}$, SE x $\mathrm{C}_{\text {org }}$ e CTC $x$ SE, respectivamente). Resultados semelhantes foram obtidos por Loux et al. (1989) e Regitano et al. (1997), exceto pelo fato de o $K_{f}$ não ter apresentado correlação com a CTC dos solos por eles estudados (TABELA 3). Isto poderia ser explicado pela diferente composição mineralógica dos solos considerados neste

TABELA 1 - Atributos químicos, físicos e mineralógicos dos solos.

\begin{tabular}{|c|c|c|c|c|c|c|c|c|c|c|c|c|c|c|c|c|c|}
\hline \multirow[t]{3}{*}{ Solo $^{\dagger}$} & \multicolumn{10}{|c|}{ Atributo químico e físico } & \multicolumn{7}{|c|}{ Atributo mineralógico§ } \\
\hline & \multirow[t]{2}{*}{$\mathrm{pH}$} & \multirow{2}{*}{$\frac{\phi_{a}}{\%}$} & \multirow{2}{*}{$\frac{C_{\text {org }}}{\mathrm{g} \mathrm{kg}^{-1}}$} & \multirow{2}{*}{$\frac{S E}{m^{2} g^{-1}}$} & \multirow{2}{*}{$\frac{\mathrm{CTC}_{\mathrm{e}}}{--\mathrm{mmo}}$} & \multirow{2}{*}{$\frac{\text { CTC }}{\mathrm{I}_{\mathrm{c}} \mathrm{kg}^{-1}-}$} & \multirow{2}{*}{$\begin{array}{l}\mathrm{V} \\
\%\end{array}$} & \multirow[t]{2}{*}{ Argila } & \multirow[t]{2}{*}{ Areia } & \multirow[t]{2}{*}{ Silte } & \multicolumn{2}{|r|}{$\mathrm{Fe}_{2} \mathrm{O}_{3}$} & \multicolumn{5}{|c|}{ Mineral de argila } \\
\hline & & & & & & & & & & & ------ & ------- & K & $\mathrm{Gb}$ & HIV & Ve & $S$ \\
\hline$R Q$ & 4,5 & 82 & 3,7 & 31,7 & 10,5 & 66,5 & 10 & 80 & 900 & 20 & 20 & (Gt) & +++ & + & + & & \\
\hline LVd & 5,4 & 98 & 18,8 & 82,5 & 28,0 & 98,0 & 29 & 220 & 700 & 80 & 70 & $(\mathrm{Hm})$ & +++ & & + & & \\
\hline Nvef & 5,9 & 99 & 27,5 & 94,9 & 132,8 & 222,8 & 60 & 550 & 250 & 200 & 250 & $(\mathrm{Hm})$ & ++++ & + & & & \\
\hline LVAd & 4,7 & 90 & 10,6 & 38,7 & 15,8 & 92,8 & 14 & 220 & 720 & 60 & 30 & $(\mathrm{Hm}+\mathrm{Gt})$ & +++ & + & & & \\
\hline LAw & 4,9 & 92 & 18,9 & 64,7 & 28,7 & 186,7 & 14 & 350 & 590 & 60 & 70 & (Gt) & ++++ & ++ & & & \\
\hline LAw' & 5,9 & 99 & 3,3 & 59,4 & 3,7 & 33,7 & 11 & 480 & 430 & 90 & 100 & (Gt) & ++++ & +++ & & & \\
\hline LVdf & 5,0 & 94 & 19,4 & 76,7 & 52,9 & 182,9 & 29 & 700 & 160 & 140 & 400 & $(\mathrm{Hm})$ & ++++ & ++ & & & \\
\hline LVwf & 4,6 & 86 & 17,1 & 65,0 & 20,7 & 192,7 & 7 & 700 & 110 & 90 & 320 & $(\mathrm{Hm})$ & ++ & +++ & & & \\
\hline LVwf' & 5,0 & 94 & 6,9 & 75,5 & 5,3 & 105,3 & 5 & 670 & 180 & 150 & 340 & $(\mathrm{Hm})$ & +++ & +++ & & & \\
\hline VEo & 5,1 & 95 & 20,3 & 119,1 & 118,5 & 237,5 & 49 & 420 & 290 & 290 & - & & ++ & & & & +++ \\
\hline GXbd & 3,9 & 57 & 78,5 & 130,5 & 112,9 & 404,9 & 6 & 570 & 130 & 300 & - & & ++++ & + & + & r & \\
\hline
\end{tabular}

† Ver Material e Métodos para nome completo dos solos

$\ddagger \phi_{\mathrm{a}}=$ porcentagem de espécies aniônicas $\left(\phi_{\mathrm{a}}=\left(1+10^{\mathrm{pH}-\mathrm{pka}}\right)^{-1}\right), \mathrm{pH}$ em CaCl $\mathrm{C}_{2} 0,005 \mathrm{~mol} \mathrm{~L}^{-1}(1: 2,5)$, $\mathrm{C}_{\text {org }}=$ carbono orgânico, SE $=$ superfície específica, $\mathrm{CTC}_{\mathrm{e}}=$ capacidade de troca de cátions efetiva, $\mathrm{CTC}=$ capacidade de troca de cátions, $\mathrm{V}=$ saturação em bases

$\S+,++++=$ relativa ocorrência dos minerais de argila: $\mathrm{K}=$ caulinita, $\mathrm{Gb}=$ gibsita, $\mathrm{Ve}=$ vermiculita, $\mathrm{S}=$ esmectita, 
trabalho, os quais apresentam elevados teores de óxidos de Fe e Al e caulinita (TABELA 1). Goetz et al. (1986) encontraram que os óxidos de $\mathrm{Fe}$ e Al (hematita e gibbsita) e a caulinita foram as principais frações do solo responsáveis pela sorção do imazaquin quando o teor de $C_{\text {org }}$ foi baixo $(<1 \%)$.

Quando os resultados relativos ao GXbd foram retirados do estudo de correlação, somente o teor de argila correlacionou-se com $\mathrm{K}_{\mathrm{f}}$ (TABELA 3). Provavelmente, isso ocorreu porque os teores de $\mathrm{C}_{\text {org }} \mathrm{e}$ de CTC total deste solo eram bem maiores e o pH bem menor que os dos demais solos (TABELA 1). Com isso, as correlações lineares do $\mathrm{K}_{f}$ com estes atributos $\left(\mathrm{C}_{\text {org }} \mathrm{e}\right.$ CTC) foram significativas somente quando a amostra do Gleissolo estava presente. Para o herbicida também aniônico primisulfuron, pertencente a classe das sulfuniluréias, a correlação entre a sorção e os teores de argila e de Al e Fe extraíveis dos solos foi igualmente positiva (Ucrainczyk \& Ajwa, 1996). Três mecanismos foram propostos por estes autores: interações hidrofóbicas, interações tipo dipolo-dipolo e troca de ânions formando complexos ("out-sphere") nos sítios com cargas dependentes de $\mathrm{pH}$.

$\mathrm{O}$ pequeno número de amostras deste estudo limita conclusões definitivas, mas parece razoável inferir que o teor de $\mathrm{C}_{\text {org }}$ e o $\mathrm{pH}$ da solução do solo influenciaram diretamente a sorção do imazaquin, enquanto que a correlação com a SE e a CTC do solo deveu-se aos efeitos indiretos do teor de $\mathrm{C}_{\text {org }}$. $O$ teor de argila correlacionou-se positivamente com a quantidade sorvida de imazaquin, principalmente em solos com teores baixos e médios de $C_{o r g}$. As superfícies planares de alguns compostos orgânicos (hidrocarbonetos aromáticos) ficaram adsorvidas diretamente às superfícies dos minerais de argila, o que comprovou a hidrofobicidade das superfícies siloxanas em esmectitas (Jaynes \& Boyd, 1991). Saada et al. (1995) observaram que o potencial hidrofóbico da caulinita é consideravelmente maior que o da ilita, o que explicou a maior sorção do asfaltene à caulinita.

Num ambiente dinâmico como o solo, não existe um único atributo capaz de ditar a sorção de pesticidas em geral. No caso do imazaquin, entretanto, as interações entre os teores de $\mathrm{C}_{\text {org }}$ e argila e os valores de $\mathrm{pH}$ devem ser consideradas, principalmente para se entender melhor seu potencial de sorção. Desta forma, estudos de regressão foram elaborados entre os valores de $\mathrm{K}_{\mathrm{f}}$ e as razões $\Delta_{1}=\mathrm{pH} / \mathrm{C}_{\text {org }}, \Delta_{2}=\mathrm{pH} /$ Argila e $\Delta_{3}=\mathrm{pH} /$ $\left(\mathrm{C}_{\text {org }} \times\right.$ Argila) para os resultados deste trabalho, somados aos de Loux et al. (1989) e de Regitano et al. (1997), totalizando 56 pontos de avaliação.

Correlação exponencial negativa foi observada entre a sorção de imazaquin e a razão entre o pH e o teor de $\mathrm{C}_{\text {org }}$ do solo $\left(\Delta_{1}\right)$, com elevado coeficiente de determinaçăo (65\%, Figura $2 \mathrm{~A})$. Quanto menor o valor do $\mathrm{pH}$ e maior o teor de $\mathrm{C}_{\text {org }}$, maior foi a sorção do imazaquim. $\mathrm{O}$ valor de $\mathrm{K}_{\mathrm{f}}$ aumentou de forma abrupta quando $\Delta_{1}<0,35$, isto é, abaixo do ponto de inflexão da curva. Este resultado seria esperado, principalmente, em função do caráter hidrofóbico do imazaquin em ambientes com baixos valores de $\mathrm{pH}$ (Stougaard et al., 1990).

A correlação entre $K_{f}$ e a razão entre $\circ \mathrm{pH}$ e o teor de argila não foi significativa $\left(\Delta_{2}\right.$, Figura $\left.2 \mathrm{~B}\right)$, ou seja, estes atributos do solo não foram suficientes para predizer a sorção do imazaquin em solos altamente intemperizados. Com base nestes resultados, avaliou-se a influência do teor de $\mathrm{C}_{\text {org }}$ em associação ao teor de argila e ao pH na sorção do imazaquin. Quando a razão $\Delta_{3}$ foi considerada como variável independente no estudo

TABELA 2 - Constantes das isotermas de Freundlich ( $\mathrm{N}=$ declividade, $\mathrm{K}_{\mathrm{f} \text { - obs }}=$ coeficiente de sorção), porcentagens sorvidas (S) e coeficientes estimados pelas equações de regressão $\left(K_{f-2 A}, K_{f-2 B}\right.$ e $\left.K_{f-2 c}\right)$.

\begin{tabular}{|c|c|c|c|c|c|c|c|}
\hline \multirow[t]{2}{*}{ Solo $^{+}$} & \multicolumn{4}{|c|}{ Isoterma de Fre undlich } & \multicolumn{3}{|c|}{ Equação de Regressão } \\
\hline & $\mathrm{N}$ & $\mathrm{r}^{2}$ & $\mathrm{~S}$ & $K_{f-o b s}$ & $\mathrm{~K}_{\mathrm{f}-2 \mathrm{~A}}$ & $\mathrm{~K}_{\mathrm{f}-2 \mathrm{~B}}$ & $K_{f-2 C}$ \\
\hline & & & $\%$ & & & & \\
\hline$R Q$ & 0,84 & 0,99 & $30,1 \pm 7,6$ & 0,36 & 0,46 & 1,10 & 0,86 \\
\hline LVd & 0,83 & 0,99 & $47,8 \pm 4,6$ & 0,49 & 1,34 & 1,10 & 0,87 \\
\hline NVef & 0,84 & 1,00 & $35,6 \pm 5,3$ & 0,69 & 2,28 & 1,36 & 2,88 \\
\hline LVAd & 0,85 & 1,00 & $33,6 \pm 3,4$ & 0,73 & 0,64 & 1,10 & 0,86 \\
\hline LAw & 0,88 & 1,00 & $24,3 \pm 2,1$ & 0,86 & 1,62 & 1,12 & 1,02 \\
\hline LAw' & 0,99 & 1,00 & $18,5 \pm 3,8$ & 1,04 & 0,46 & 1,18 & 0,85 \\
\hline LVdf & 0,86 & 1,00 & $53,2 \pm 4,1$ & 1,17 & 1,64 & 5,33 & 3,22 \\
\hline LVwf & 0,89 & 1,00 & $52,2 \pm 3,2$ & 1,71 & 1,51 & 7,72 & 2,96 \\
\hline LVwf $^{\prime}$ & 0,90 & 1,00 & $47,9 \pm 2,3$ & 3,00 & 0,47 & 4,40 & 0,88 \\
\hline VEo & 0,83 & 1,00 & $52,4 \pm 3,9$ & 1,99 & 1,72 & 1,19 & 1,30 \\
\hline GXbd & 0,84 & 1,00 & $82,1 \pm 2,0$ & 19,29 & 10,01 & 6,45 & 19,24 \\
\hline
\end{tabular}

† Ver Material e Métodos para nome completo dos solos.

$\ddagger \mathrm{K}_{\mathrm{f}-2 \mathrm{~A}}, \mathrm{~K}_{\mathrm{f}-2 \mathrm{~B}}, \mathrm{~K}_{\mathrm{f}-2 \mathrm{C}}=\mathrm{K}_{\mathrm{f}}$ calculado pelas equações das Figuras $2 \mathrm{~A}, 2 \mathrm{~B}$ e $2 \mathrm{C}$, respectivamente. 
de regressão, houve diminuição no desvio dos pontos em relação ao modelo da Figura $2 \mathrm{~A}$, sendo a interação entre esses atributos capaz de explicar $85 \%$ da variância (Figura 2C). Isto representa $20 \%$ a mais do que quando o teor de argila não foi incluído no modelo (Figura 2A), o que reforça a importância da granulometria do solo na sorção do imazaquin. Loux et al. (1989) também observaram que a precisão do modelo de regressão diminuiu em $18 \%$ quando o teor de argila foi excluído. $\mathrm{Na}$ maioria dos casos, o aumento na sorção do imazaquin com o aumento nos teores de $\mathrm{C}_{\text {org }}$ e/ou argila tornou-se mais pronunciado (ou seja, $\Delta_{3}<0,005$ ), quando o valor do $\mathrm{pH}$ da solução do solo foi menor que 5,5 (Figura 2C).

TABELA 3 - Coeficientes de correlação linear $(r)$ entre $K_{f} e$ alguns atributos dos solos.

\begin{tabular}{lcccc}
\hline $\begin{array}{l}\text { Atributo } \\
\text { do Solo }\end{array}$ & $\begin{array}{c}\text { Trabalho } \\
\text { Atual }\end{array}$ & $\begin{array}{c}\text { Atual } \\
\text { (sem o } \\
\text { GXbd) }\end{array}$ & $\begin{array}{c}\text { Loux et al. } \\
(1989)\end{array}$ & $\begin{array}{c}\text { Regitano } \\
\text { et al. } \\
(1997)\end{array}$ \\
\hline C $_{\text {org }}$ & $0,91^{* *}$ & $-0,11$ & $0,45^{*}$ & $0,74^{* *}$ \\
Argila & 0,27 & $0,65^{*}$ & 0,21 & 0,09 \\
CTC & $0,78^{* *}$ & 0,22 & 0,35 & 0,11 \\
SE & $0,64^{*}$ & 0,40 & & - \\
pH & $-0,62^{*}$ & $-0,12$ & $-0,59^{* *}$ & $-0,56^{*}$ \\
\hline
\end{tabular}
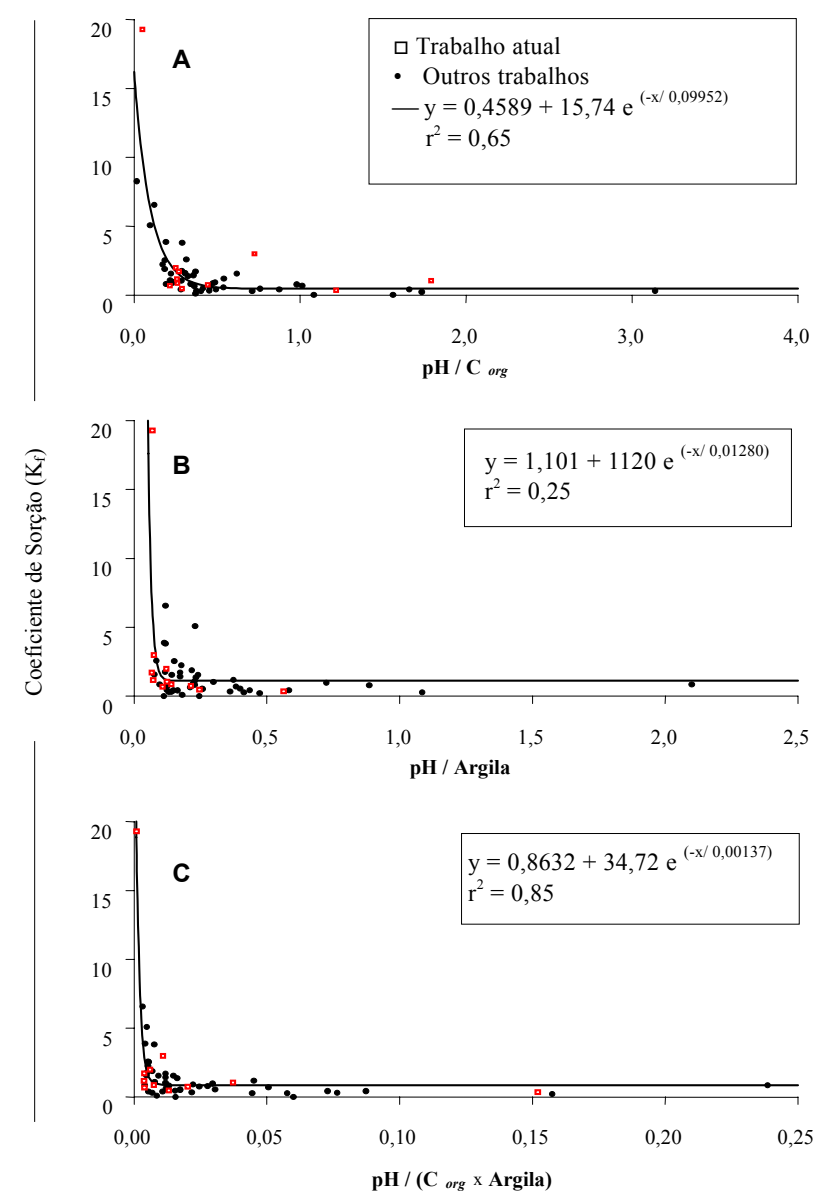

Figura 2 - Estudos de regressão entre o coeficiente de sorção $\left(K_{f}\right)$ do imazaquin e a razão entre alguns atributos do solos: (A) $\Delta_{1}=\mathrm{pH} / \mathrm{C}_{\text {org }}$, (B) $\Delta_{2}=\mathrm{pH} /$ Argila, (C) $\Delta_{3}=\mathrm{pH} /\left(\mathrm{C}_{\text {org }} \times\right.$ Argila $)$.

\section{CONCLUSÕES}

A sorção do imazaquin variou de baixa a moderada nos solos estudados, exceto no $\mathrm{GH}$. O teor de $\mathrm{C}_{\text {org }}$ e o $\mathrm{pH}$ da solução foram os principais atributos do solo correlacionados com a sorção do imazaquin. A inclusão do teor de argila na análise de regressão aumentou em $20 \%$ a precisão do modelo. O imazaquin apresenta maior disponibilidade e, portanto, maior potencial de lixiviação em solos com baixos teores de $\mathrm{C}_{\text {org }}$ e argila e com valores de $\mathrm{pH}$ acima de 6,0.

\section{AGRADECIMENTOS}

À FAPESP, pelo financiamento da pesquisa (Processo 98/09614-7). Ao CNPq, pelo apoio dado à elaboração da pesquisa, por meio da concessão de Bolsa de Jovem Pesquisador à primeira autora (Processo 301160/93-7).

\section{REFERÊNCIAS BIBIOGRÁFICAS}

ALI, M.A.; DZOMBAK, D.A. Competitive sorption of simple organic acids and sulfate on goethite. Environmental Science and Technology, v.30, p.1061-1071, 1996.

BASKARAN, S.; BOLAN, N.S.; RAHMAN, A.; TILLMAN, R.W. Pesticide sorption by allophanic and non-allophanic soils of New Zealand. New Zealand Journal of Agricultural Research, v.39, p.297-310, 1996.

BHALLA, P.; HACKETT, N.M.; HART, R.G.; LIGNOWSDI, M. Imazaquin herbicide. In: SHANER, D.L.; O'CONNOR, S.L. (Ed.) The imidazolinone herbicides. Boca Raton: CRC Press, 1991. p.237-245.

CAMARGO, O.A.; MONIZ, A.C.; JORGE, J.A.; VALADARES, J.M.A.S. Métodos de análise química, mineralógica e física de solos do Instituto Agronômico de Campinas. Campinas: IAC, 1986. 94p. (Boletim Técnico, 106).

CIHACEK, J.L.; BREMNER, J.M. A simplified ethylene glycol monoethyl procedure for assessment of soil surface area. Soil Science Society of America Journal, v.43, p.821-822, 1979.

EVANKO, C.R.; DZOMBAK, D.A. Influence of structural features on sorption of NOM-analogue organic acids to goethite. Environmental Science and Technology, v.32, p.2846-2855, 1998.

GOETZ, A.J.; WEHTJE, G.; WALKER, R.H.; HAJEK, B. Soil solution and mobility characterization of imazaquin. Weed Science, v.34, p.788-793, 1986.

GROVER, R. Adsorption of picloram by soil colloids and various other adsorbents. Weed Science, v.19, p.417-418, 1971.

HASSET, J.J.; BANWART, W.L.; WOOD, S.G.; MEANS, J.C. Sorption of $\alpha$-naphthol: Implications concerning the limits of hydrophobic partitioning. Soil Science Society of America Journal, v.45, p.38-42, 1981.

HUANG, P.M.; WANG, T.S.C.; WANG, M.K.; WU, M.H.; HSU, N.W. Retention of phenolic acids by noncrystalline hydroxyaluminum and -iron compounds and clay minerals of soils. Soil Science, v.123, p.213-219, 1977

JAYNES, W.F.; BOYD, S.A. Hydrophobicity of siloxane surfaces in smectites as revealed by aromatic hydrocarbon adsorption from water. Clays and Clay Minerals, v.39, p.428-436, 1991.

KHAN, A.; HASSET, J.J.; BANWART, W.L.; MEANS, J.C.; WOOD, S.G. Sorption of acetophenone by sediments and soils. Soil Science, v.128, p.297-302, 1979. 
KOSKINEN, W.C.; HARPER, S.S. The retention process: mechanisms. In: CHENG, H.H.; BAILEY, G.W.; GREEN, R.E.; SPENCER, W.F. (Ed.) Pesticides in the soil environment: Processes, impacts, and modeling. Madison: SSSA, 1990. p.51-77.

LOUX, M.M.; LIEBL, R.A.; SLIFE, F.W. Adsorption of imazaquin and imazethapyr on soils, sediments, and selected adsorbents. Weed Science, v.37, p.712-718, 1989.

NICHOLLS, P.H.; EVANS, A.A. Sorption of ionisable organic compounds by field soils. Pesticide Science, v.33, p.319330, 1991.

RATNER-ZOMAR, Y.; BANIN, A.; CHEN, Y. Oven drying as a pretreatment for surface area determinations of soils and clays. Soil Science Society of America Journal, v.47, p.1056-1058, 1983.

REGITANO, J.B.; BISCHOFF, M.; LEE, L.S.; REICHERT, J.M.; TURCO, R.F. Retention of imazaquin in soil. Environmental Toxicology and Chemistry, v.16, p.397-404, 1997.

REGITANO, J.B.; ALLEONI, L.R.F.; VIDAL-TORRADO, P.; CASAGRANDE, J.C.; TORNISIELO, V.L. Imazaquin sorption in highly weathered tropical soils. Journal of Environmental Quality, v.29, p.894-900, 2000.
SAADA, A.; SIFFERT, B.; PAPIRER, E. Comparisson of the hydrophilicity/ hydrophobicity of illites and kaolinites. Journal of Colloid and Interface Science, v.174, p.185-190, 1995.

SANNINO, F.; VIOLANTE, A.; GIANFREDA, L. Adsorptiondesorption of 2,4-D by hydroxy aluminum montmorillonite complexes. Pesticide Science, v.51, p.429-435, 1997.

STEVENSON, F.J. Organic matter reactions involving pesticides in soil. In: KAUFMAN, D.D.; STILL, G.G.; PAULSON, G.D.; BANDAL, S.K. (Ed.) Bound and conjugated pesticide residues. Washington, DC: American Chemistry Society, 1976. p.180-207. (ACS Symposium Series, 29).

STOUGAARD, R.N.; SHEA, P.J.; MARTIN, A.R. Effect of soil type and $\mathrm{pH}$ on adsorption, mobility, and efficacy of imazaquin and imazethapyr. Weed Science, v.38, p.67-73, 1990.

UKRAINCZYK, L.; AJWA, H.A. Primisulfuron sorption on minerals and soils. Soil Science Society of America Journal, v.60, p.460-467, 1996.

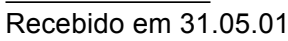

